\title{
Proof of Principle that Molecular Modeling Followed by a Biophysical Experiment Can Develop Small Molecules that Restore Function to the Cardiac Thin Filament in the Presence of Cardiomyopathic Mutations
}

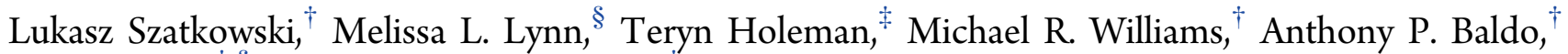 \\ Jil C. Tardiff,, $\$$ and Steven D. Schwartz*, ${ }^{\ddagger}$ \\ ${ }^{\dagger}$ Department of Chemistry and Biochemistry, University of Arizona, Tucson, Arizona 85721, United States \\ ${ }^{\ddagger}$ Department of Physiological Sciences and ${ }^{\S}$ Department of Medicine, University of Arizona, Tucson, Arizona 85724, United States \\ Supporting Information
}

ABSTRACT: This article reports a coupled computational experimental approach to design small molecules aimed at targeting genetic cardiomyopathies. We begin with a fully atomistic model of the cardiac thin filament. To this we dock molecules using accepted computational drug binding methodologies. The candidates are screened for their ability to repair alterations in biophysical properties caused by mutation. Hypertrophic and dilated cardiomyopathies caused by mutation are initially biophysical in nature, and the approach we take is to correct the biophysical insult prior to irreversible cardiac damage. Candidate molecules are then tested experimentally for both binding and biophysical properties. This is a proof of concept study-eventually candidate molecules will be tested in transgenic animal models of genetic (sarcomeric) cardiomyopathies.

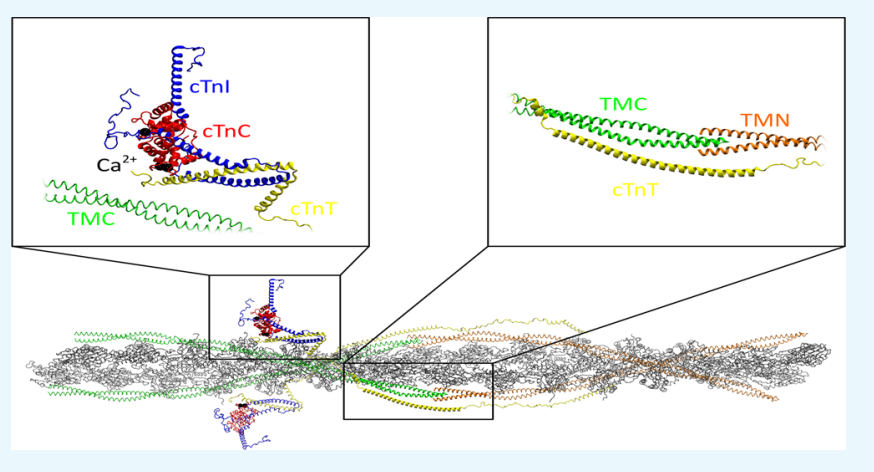

\section{INTRODUCTION}

This paper describes a recently developed approach to identify drug candidates that are able to ameliorate the effects of disease-causing mutations in the cardiac thin filament (CTF). This complex multi-component system is largely responsible for the regulation of cardiac contraction and relaxation. Point mutations in this component of the cardiac sarcomere are well known to cause both hypertrophic and dilated cardiomyopathies in patients. ${ }^{1-3}$ At present, treatment of these diseases is largely limited to symptom palliation. Biophysical understanding of drug action in these diseases and heart failure is in the early stages. One provocative example is the study of omecamtiv mecarbil. ${ }^{4}$ Here, the drug was determined to have almost the opposite effect on the thick filament myosin as was expected. One other case of a molecularly targeted drug is MYK-461 that is designed to effect sarcomere energetics. Targeted drug therapies have proven difficult to design for genetic cardiomyopathies given the long-time course of the disease development (often decades). Modification thus must be brought about by biophysical changes at the level of the sarcomere. The approach described in this paper employs a recently described all atom model of the CTF (Figure 1) that allows exposure of biophysical insults to the CTF; ${ }^{7,8}$ methods to screen and dock potential drug candidates followed by all atom molecular dynamics simulations to check for repair of the damage to function. We experimentally validate our computational results using isothermal titration calorimetry (ITC) and differential scanning calorimetry (DSC). This in silico followed by an in vitro approach will eventually be followed by transgenic in vivo animal studies, and we feel this paradigm represents an unexplored approach to the development of treatment for an unmet need in a complex but not uncommon disorder. The approach is viable for any of the hundreds of mutations in the CTF that cause hypertrophic cardiomyopathy and dilated cardiomyopathy . In addition, the initial computational phases of the approach are fairly high throughput and essentially no cost.

We will describe the application of the methodology to a specific mutation in the TM component of the CTF. An important region of the CTF is the TNT1 domain of cTnT and overlap of the C-terminal end of one TM and N-terminal end of the next TM. ${ }^{7,9,10}$ It modulates the cooperative activation of the myofilament. One of the known human cardiomyopathic mutations linked to DCM within this region is the TM Asp230Asn (D230N) mutation. This mutation causes a compaction of the cTnT-TM overlap region

Received: November 29, 2018

Accepted: March 28, 2019

Published: April 9, 2019 


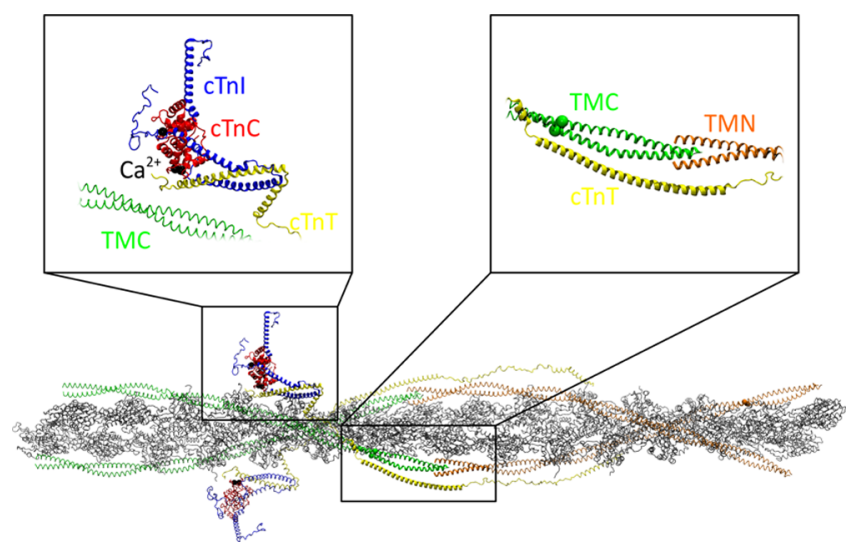

Figure 1. Structure of the full atomistic CTF model. F-actin coregray/silver; tropomyosin (TM)-green/orange; troponin C $(\mathrm{cTnC})$-red; troponin I ( $\mathrm{cTnI})$-blue; troponin $\mathrm{T}(\mathrm{cTnT})-$ yellow; and black spheres-Ca ions. The drug binding pocket within the $\mathrm{cTnC}$ subunit and $\mathrm{cTnT}-\mathrm{TM}$ overlap region that will be crucial to this work are shown in the overall figure and in expanded versions above. The mutation site on TM is indicated as green balls.

accompanied by an increased cooperativity (decreased flexibility) of the Tm filament. ${ }^{7,10,11}$ A detailed description of the TM D230N mutation from molecular dynamics studies and experiments has been previously presented. ${ }^{7,10}$ (We note that the computational and experimental model are both chosen to have mutant amino acid residues in both chains of the TM dimer. This is done to create interpretable experimental results and then to match the calculations to the experimental model). Various studies have shown that structural effects of mutations within the CTF can propagate over long distances and cause changes in the global flexibility and CTF structure. ${ }^{8,12-14}$ Therefore, one should ask if drugs used to treat cardiomyopathies also could have similar influence over long distances from a drug binding site on the dynamic and structural properties of the CTF.

The mechanism of drug binding to the CTF remains largely opaque. Past studies of drug-modulated calcium binding affinities show that drugs bind within the cTnC subunit. ${ }^{15-27}$ The most commonly used models in the experimental and theoretical approaches include only residues of $\mathrm{N}$-lobe of the cTnC subunit (residues 1-90) with a small representation of the inhibitory region of the cTnI subunit (residues 144-163). The use of fragments to study drug binding can be misleading as local models do not allow the prediction of the effects of drugs on the dynamic and structural nature of the CTF over long distances. Such "allosteric action at a distance" has been central in our published work to detect potential "molecular off target” effects. In the current study, unrestricted search throughout the entire thin filament has allowed us to locate a new drug-binding pocket within the core region of the cTn complex. Again, the most likely binding site is found in the $\mathrm{TnC}$ core region. This is then followed by detailed ligand docking studies, molecular dynamics analysis of the effects of drug binding on the mutant thin filament, and finally in vitro experimental evaluation of potential new drugs.

\section{RESULTS}

Binding locations evaluated using the Schrödinger SiteMap ${ }^{28}$ program revealed a new binding pocket between $\mathrm{N}$-lobe and C-lobe of the cTnC subunit. The Schrödinger Glide ${ }^{29}$ program was used to perform docking studies for variety of ligands from ZINC15 database ${ }^{30}$ to the binding pocket between the $\mathrm{N}$ and $\mathrm{C}$ lobes of cTnC (for details see Experimental Materials and Methods section). As a result of the initial docking studies of the TM D230N mutant, a set of eight ligands with the best average Glide extra precision (XP) docking scores were chosen for the initial molecular dynamics (MD) trials. The first metric employed to test for putative drug effectiveness was the distance in the overlap region between the cTnT and TM chains. As reported previously, this corresponds to the average distance between residues 94-133 of cTnT and the center of the TM coiled coil. ${ }^{7}$ A candidate drug is thus identified as one that restores the overlap distance in a mutant to be similar to that of the WT. This winnowed the number of tested ligands to three. The code names and the structures of the final three ligands, their names, and docking scores are presented in Figure 2. We initially computed the average distances in the cTnT-TM overlap region. This was computed as a distance of the centers of mass of the helices of TM and TnT. Errors are calculated as the standard error of the means. The WT average distance is $19.9 \pm 0.2 \AA$, and the D230N CTF without drug was previously found to be $18.5 \pm 0.1 \AA^{7}$ The results with drug candidates bound are $19.0 \pm 0.2,21.6 \pm 0.3$, and $20.1 \pm 0.1 \AA$, respectively, for the TM D230N systems with the Z01, Z06, and Z08 ligands. Thus, Z01 was found to only partially relieve the compaction in the overlap region that results in the disease state, but we used it to test our binding predictions experimentally, employing ITC to directly measure the binding affinity $\left(K_{d}\right)$ and enthalpy $(\Delta H)$ of $Z 01$ in vitro. ITC is a highly sensitive assay for the characterization of the fundamental driving forces of molecular binding reactions. Given the similarity in the predicted docking scores for the

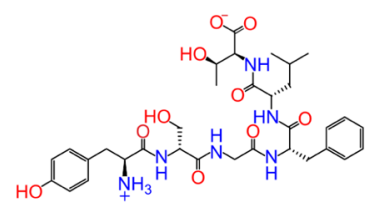

201

2-Ser-Thr-Leu-enkephalin Tyr-D-Ser-Gly-Phe-Leu-Thr

D230N $\quad-9.6$ WT $\quad-9.1$

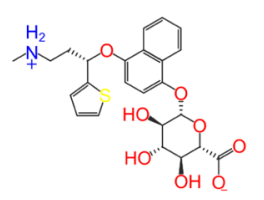

206

4-Hydroxy Duloxetine $\beta$-DGlucuronide

$-10.3$

$-8.6$

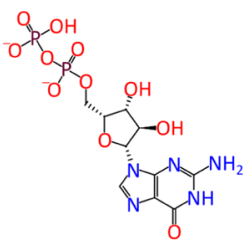

Z08

Guanosine 5'-diphosphate

$-9.5$

$-9.3$

Figure 2. Three ligands chosen after initial tests according to cTnT-TM overlap distances. From the top to bottom: structure of ligands with their ionic states predicted by Schrödinger LigPrep, code names, full names, and average docking scores to the TM D230N mutant and to the WT. 

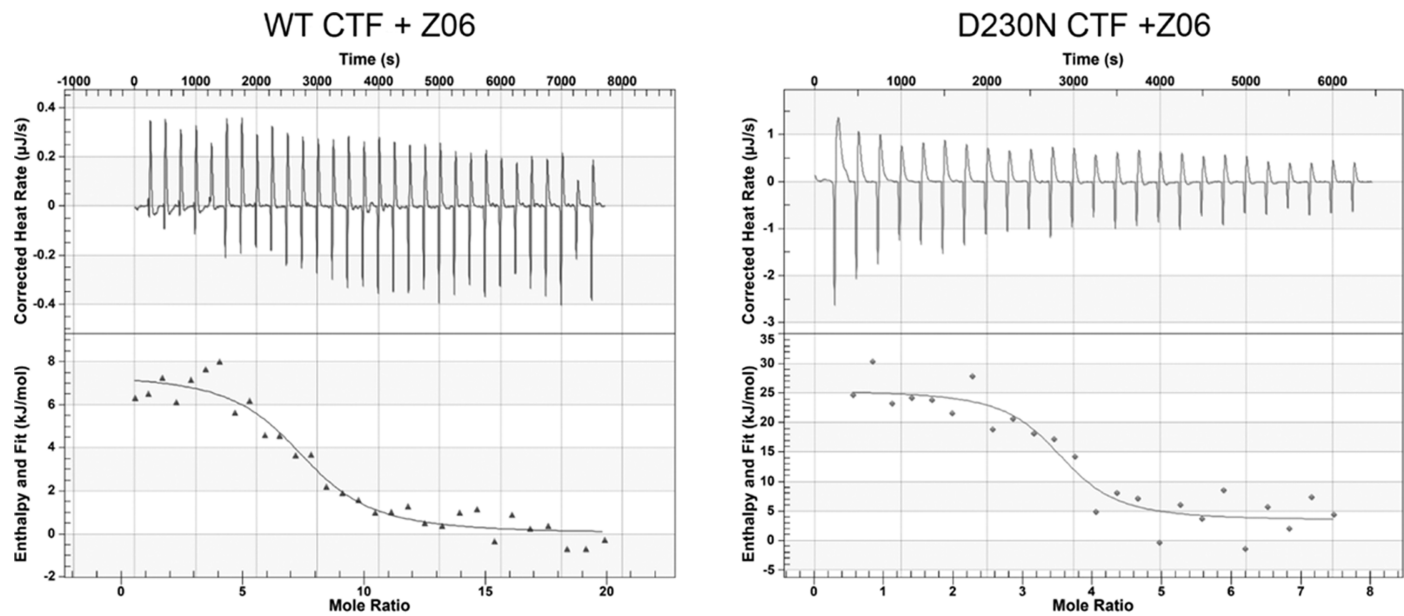

WT CTF + Z01

D230N CTF + Z01
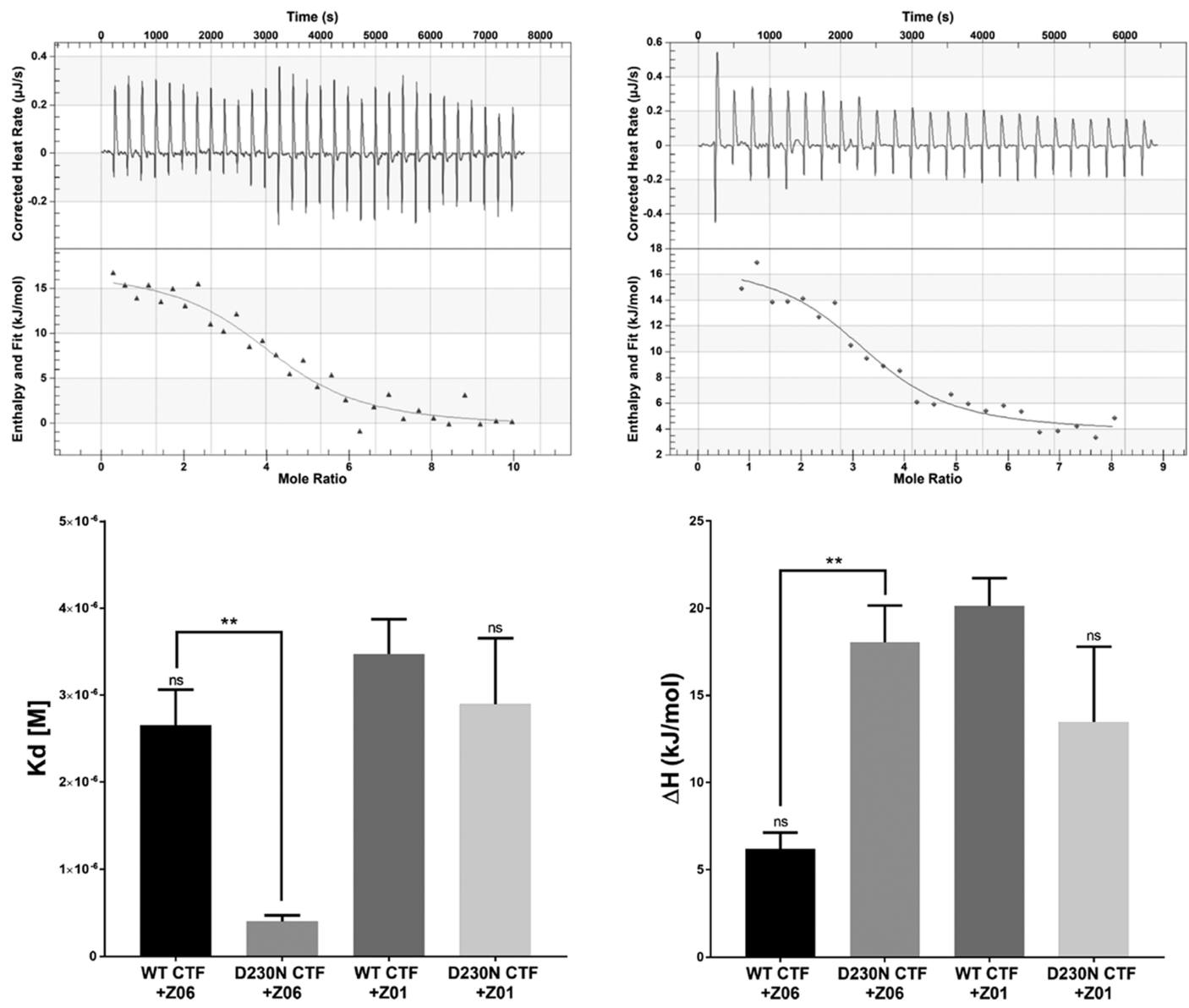

Figure 3. ITC performed on both the wildtype and D230N mutant with two candidate ligands bound. The top panels show both the raw data and the calculated enthalpy of binding, and the bottom panels show the extracted binding constants.

Z01 compound, we expected there to be little difference in its binding affinity in vitro for either the WT or D230N CTF (Figure 2). Indeed, as our model predicted, there was no significant difference in the binding affinity of $Z 01$ to the WT $(2.9 \pm 0.8 \mu \mathrm{M})$ or $\mathrm{D} 230 \mathrm{~N}(3.5 \pm 0.4 \mu \mathrm{M}) \mathrm{CTF}$ (Figure 3 and Table 1). Thus, while $Z 01$ did not meet the criteria of alleviating TM/TnT helix compaction, it was included in our ITC tests as a way to verify the accuracy of the binding algorithm. Note that the enthalpy is reported as positive-this measures the heat deposited in the environment, the negative of the binding enthalpy.

We thus are left with two candidate molecules (Z06 and Z08). First, we evaluated the Z08 ligand because for this system, we predicted an overlap distance closer to the WT than in the case of the TM D230N-Z06. An important test however is to evaluate the effect of the drug on the wildtype CTF. The vast majority of patients with disease-causing mutations are heterozygous with differing degrees of mutant protein expressed. Thus, potential deleterious effects on the WT 
Table 1. Binding Affinities Calculated via ITC ${ }^{a}$

\begin{tabular}{llc} 
& \multicolumn{1}{c}{$K_{\mathrm{d}}(\mu \mathrm{M})$} & $\Delta H, \mathrm{~kJ} / \mathrm{mol}$ \\
WT CTF + Z06 & $2.7 \pm 0.57$ & $6.2 \pm 0.93$ \\
D230N CTF + Z06 & $0.4 \pm 0.41^{* *}$ & $18.1 \pm 2.1^{* *}$ \\
WT CTF + Z01 & $3.5 \pm 0.40$ & $20.1 \pm 1.6$ \\
D230N CTF + Z01 & $2.9 \pm 0.75$ & $13.5 \pm 4.3$
\end{tabular}

${ }^{a_{\text {The }}}$ binding affinity $\left(K_{\mathrm{d}}, \mu \mathrm{M}\right)$ and Enthalpy $(\Delta H, \mathrm{~kJ} / \mathrm{mol})$ are reported for TM WT and TM D230N reconstituted thin filaments with Z06. Mean values reported \pm standard error of the mean (SEM) with an $N=4-5$ per group. A student's $t$-test was used to assess significance, $* * p<0.01$ vs WT CTF $+Z 06$.

protein that comprise the CTF must be considered. Performing this test, we found the average overlap distance for the WTZ08 system (17.0 ̊) to be even further decreased than for the untreated TM D230N mutant itself. Based on these observations, the Z08 ligand was excluded from further investigation. For the WT-Z06 system, simulation predicts an average cTnT-TM overlap distance of $19.5 \AA$, which is almost identical to the WT system. In other words, unlike the Z08 molecule, the WT is largely unaffected by Z06. Additionally, predictions of the average distances between cTnT and TM were confirmed by detailed analysis of the cTnT-TM distances through the trajectories (see Supporting Information Figures S6-S8). The significant differences in the docking scores for the Z06 ligand between the TM D230N mutant and the WT systems suggest that the Z06 ligand should bind much more strongly to the TM D230N mutant than to the WT. All computational predictions lead us to the consistent conclusions that Z06 was the best choice for the experimental trials. Therefore, the binding affinity of Z06 to WT and D230N CTF was evaluated experimentally using ITC. As the CTF docking studies predicted, Z06 bound to D230N CTF with a significantly higher affinity compared to WT $\left(K_{d}: 0.4 \pm 0.07\right.$ $\mu \mathrm{M}$ vs $2.7 \pm 0.4 \mu \mathrm{M}$, respectively). Additionally, D230N CTF binding to Z06 also had a significantly higher enthalpy $(\Delta H)$ when compared to WT CTF binding Z06 $(18.1 \pm 2.0 \mathrm{~kJ} / \mathrm{mol}$ vs $6.2 \pm 0.9 \mathrm{~kJ} / \mathrm{mol}$ respectively) (Figure 3 and Table 1 ). The Z06 ligand is one of the metabolites of duloxetine, an antidepressant drug. It is known that this molecule is not a biologically active compound and does not bind to the neurotransporters that are the targets of the parent molecule: serotonin, norepinephrine, and dopamine. ${ }^{31}$

Figure 4 shows the position of the Z06 ligand in the TM D230N and the WT systems. Differences in Z06 placement within the binding pocket are caused by differences in the side chain position for the cTn residues. Of note, Z06 does not stay stably bound in the "mutant induced site" in the absence of mutation giving credence to the fact that this specific binding is caused by mutation. On the other hand, upon mutation, the molecule is bound stably in the indicated site for as long as we have ever computed. Also, as a final test of the validity of our binding site identification, we performed ITC on the binding of $Z 06$ to the troponin complex alone. The binding constant was almost the same [ $K$ was within the experimental error of that for the full thin filament $(2.13 \pm 0.26 \mu \mathrm{M})]$. Figure 5 shows a comparison of the average structure over all trajectories for the TNT1/TM overlap region for both the treated and untreated systems. For the TM D230N mutant, the TNT1 region is both closer to the TM and its N-terminal end is shifted slightly so as to be above the TM chain. The end result is the decrease in the distance between the chains. In the
D230N

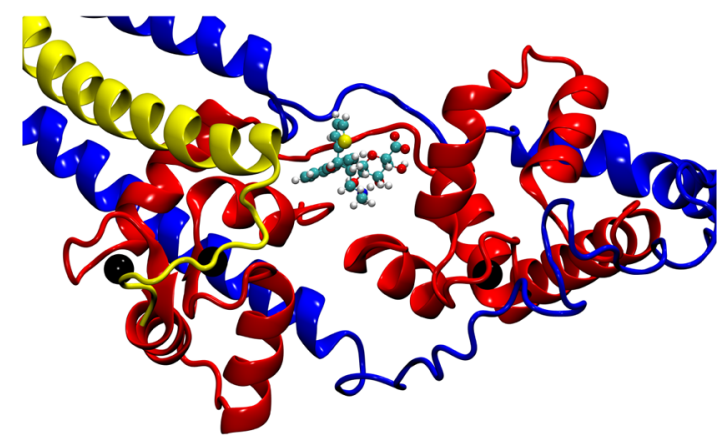

WT

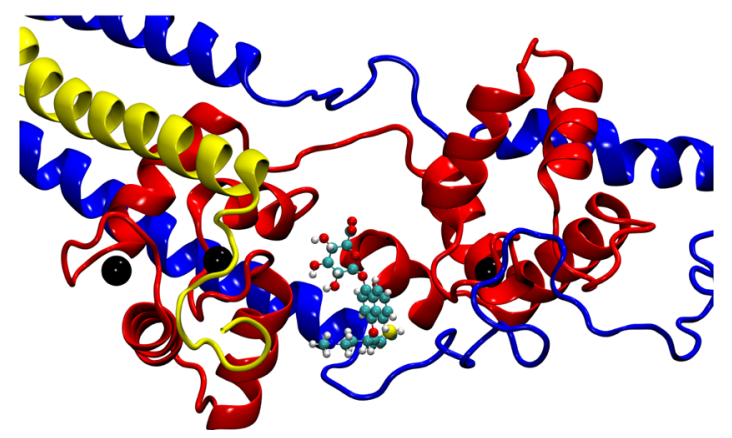

Figure 4. Position of the Z06 ligand within newly found binding pocket of the TM D230N mutant (top) and the WT (bottom). CTF ribbons: red-cTnC, blue-cTnI, yellow-cTnT; and black spheres- $\mathrm{Ca}^{2+}$ cations. Ligand representation as balls and sticks: white, cyan, blue, red, and yellow balls, respectively, denote $\mathrm{H}, \mathrm{C}, \mathrm{N}$, $\mathrm{O}$, and $\mathrm{S}$ atoms.

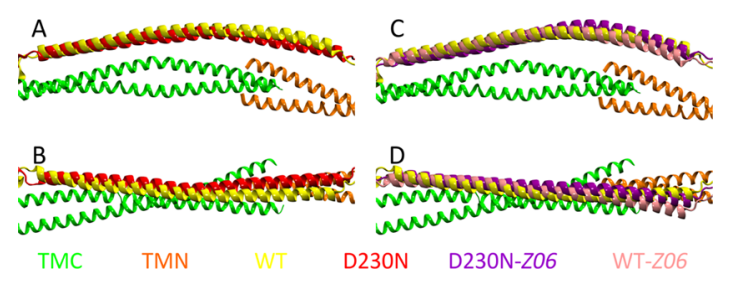

Figure 5. Comparison of the cTnT-TM overlap region in the average structures of TM D230N mutant [panels (A,B)] and TM D230N-Z06 and WT-Z06 systems [panels $(\mathrm{C}, \mathrm{D})]$ vs the WT. Panels $(\mathrm{A}, \mathrm{C})$ : a view from the side of TM chain and panels $(\mathrm{B}, \mathrm{D})$ : view with $\mathrm{TM}$ chain directly below cTnT. TM is shown from the WT structure where other structures were aligned to the WT TM chain. TM-green (Cend)/orange (N-end); WT cTnT-yellow; TM D230N cTnT-red; TM D230N-Z06 cTnT-purple; and WT-Z06 cTnT-pink.

TM D230N-Z06 system, the N-terminal end of TNT1 is slightly shifted in the opposite direction as the D230N mutant alone as compared to WT, and at the same time, we see an increase in curvature in TNT1 (above C-terminal end of TM). This change in the position of TNT1 yields a clear explanation as to why the average distance between $\mathrm{cTnT}$ and TM is larger for the TM D230N-Z06 system than in the untreated mutant. Importantly, most of the TNT1 region of the WT-Z06 is in almost an identical position as that of the WT. We emphasize that these are subtle changes-more drastic changes induced by mutation would not be compatible with life and would be 
expected to be embryonic lethal rather than the cause of a disease that takes decades to develop.

Root mean square fluctuation (RMSF) values of $\mathrm{C} \alpha$ atoms for tropomyosin chains within the overlap region shows significant changes in the flexibility of the region in the D230N mutant versus the WT (see Figure 6). Previous experiments

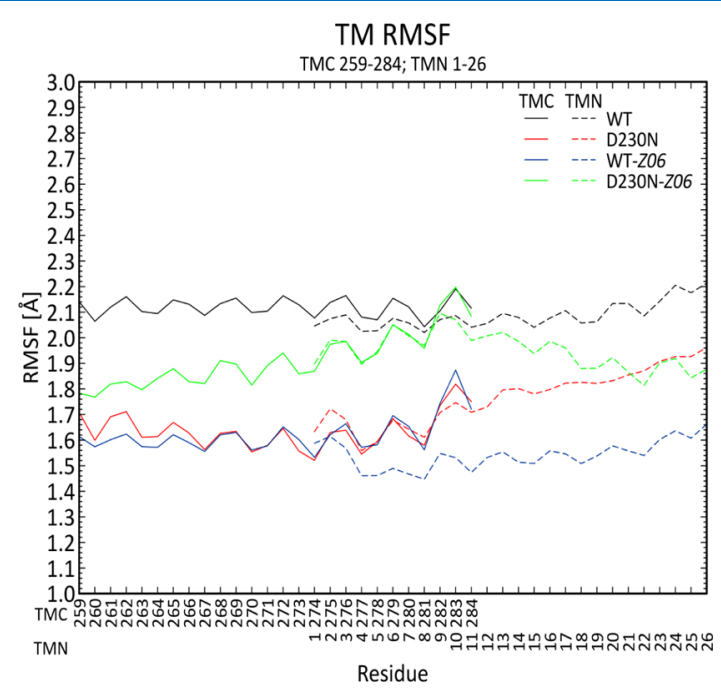

Figure 6. $\mathrm{C} \alpha$ RMSF values for 11 overlapping TMC-TMN residues plus 15 additional residues close to the TMC-TMN overlapping region for WT, TM D230N mutant, and TM D230N-Z06 and WTZ06 systems.

have shown a significant increase in the cooperativity of the Cend of the tropomyosin chain-N-end of the tropomyosin chain (TMC-TMN) region for the TM D230N mutant ${ }^{7,10}$ that correlates well with the theoretical prediction of lower flexibility of this region. Z06 ligand interactions with TM $\mathrm{D} 230 \mathrm{~N}$ mutant were able to restore a more native flexibility of the TMC-TMN overlap region to the WT level. Z06 interactions with the WT caused drop in flexibility of this region to the level from TM D230N mutant. Nevertheless, given that the Z06 affinity is much higher for the TM D230N mutant than for the WT, the Z06 ligand should have a minimal effect on WT thin filaments. We have also tested binding of the Z06 molecule independently via ITC to the troponin complex alone (i.e. no actin and no tropomyosin) and to the full thin filament. The binding constants were within experimental error, indicating that the molecule does not bind strongly to either tropomyosin or to actin, and this gives further credence that it binds in the only site identified-TnC.

We coupled these in silico data to the experiment via DSC to determine the effect of bound Z06 on the thermal stability and cooperativity (flexibility) of the CTF. (Note that lower flexibility in this region translates to stiffer mechanical movement and stronger transmission of the binding signal. This in turn is found experimentally via non-linear $\mathrm{Ca}^{2+}$ versus force generation in isolated cardiac muscle fiber studies.) An advantage of DSC for the study of the CTF is its sensitivity in detecting the calorimetric events associated with unfolding of well-characterized regions of the CTF including: the denaturation of the individual termini of TM (TMN and TNC), the TM-troponin (cTn) complex, cTn alone, as well as actin unfolding. ${ }^{3,4,32-34}$ The literature cited shows that each event has a unique independent thermal signature allowing an unambiguous assignment of specific unfolding events to specific components of the thin filament. This was checked via melting studies of individual components of the thin filament. Recently, the effect of the TM D230N mutation on the thermal stability $\left(T_{\mathrm{m}}\right)$ of the $\mathrm{N}$ - and C-termini (TMN and TMC, respectively) as well as its effect on the stability and cooperativity (full-width half-max, fwhm, a surrogate of flexibility) of the fully reconstituted CTF was published. ${ }^{7,10}$ Consistent with these data, our results show that TM D230N increases the thermal stability $\left(T_{\mathrm{m}}{ }^{\circ} \mathrm{C}\right)$ and increases the cooperativity (decreased fwhm) of domain 2 (D2) of the thermal profile, an unfolding domain that corresponds to the thermal denaturation of the TM-cTn complex from actin (Figure 7 and Table 2). Specifically, we found that the thermal stability was $46.5 \pm 0.09$ TM D230N versus $46.2 \pm 0.08$ for TM WT and the fwhm of TM D230N was decreased to $1.5 \pm$ 0.06 from $1.9 \pm 0.04{ }^{\circ} \mathrm{C}$. This confirms that the baseline effect of the mutation is to decrease the flexibility of the crucial TM overlap.

We expanded upon this to assess the effect of $Z 06$ binding to both WT and TM D230N CTF. Of note, our data indicated that $Z 06$ increases the thermal stability of D2 for both TM WT $\left(46.6 \pm 0.05{ }^{\circ} \mathrm{C}\right)$ and TM D230N $\left(46.9 \pm 0.07{ }^{\circ} \mathrm{C}\right)$ above that of unbound TM WT. This effect is explained by a well-known phenomena in which protein thermal stability is increased by ligand binding due to the equilibrium of binding and unfolding. ${ }^{35}$ Interestingly, despite this ubiquitous increase in thermal stability, the fwhm of TM-cTn unfolding (a surrogate of flexibility) for TM D230N-Z06 was significantly increased (decreased cooperativity) from TM D230N levels to $1.8 \pm$ $0.04{ }^{\circ} \mathrm{C}$, indicating a significantly less rigid (or more flexible) TM filament when Z06 is bound to TM D230N (Figure 7 and Table 2). While this is not a complete recovery of flexibility to WT levels, it is an encouraging result that agrees with our RMSF-predicted changes in the flexibility of the TM-overlap region (Figure 6). Such improvement in the flexibility of the $T_{\mathrm{m}}$ filament could positively impact $T_{\mathrm{m}}$ regulatory function, thus improving cardiac function.

\section{CONCLUSIONS}

The treatment of genetic cardiomyopathies presents a major unmet challenge in cardiac pharmacology. This paper presents a new approach to the screening and eventual development of such treatment modalities. Central to the approach is a direct connection of new theoretical and experimental approaches. The molecules we present here are provided as proof of concept. One ligand was found to largely relieve compaction in the TM overlap region associated with increased CTF cooperativity and the eventual development of DCM. In addition, this candidate molecule was shown to significantly relieve the loss of flexibility in the TM overlap region also indicated in the development of mutation induced DCM.

The Z06 ligand acts as a bridge between cTnC and cTnI residues in addition to restoring the $\mathrm{TM}-\mathrm{cTnT}$ interhelix distances. Thus, this ligand has the potential to restore the ability to transmit the calcium-binding signal from the $\mathrm{cTnC}$ subunit to the other parts of the CTF system both by modulating cooperativity and tuning of the cTnI functionality. It is only fair to discuss the limitations of the approach. This methodology is focused on the thin filament. We are proposing a method to hunt for drugs that treat that piece of the cardiac machinery. It is entirely possible that the drugs found in this way will bind elsewhere in the many thousands of proteins in a human body and have unwanted side effects. Of course, this is 

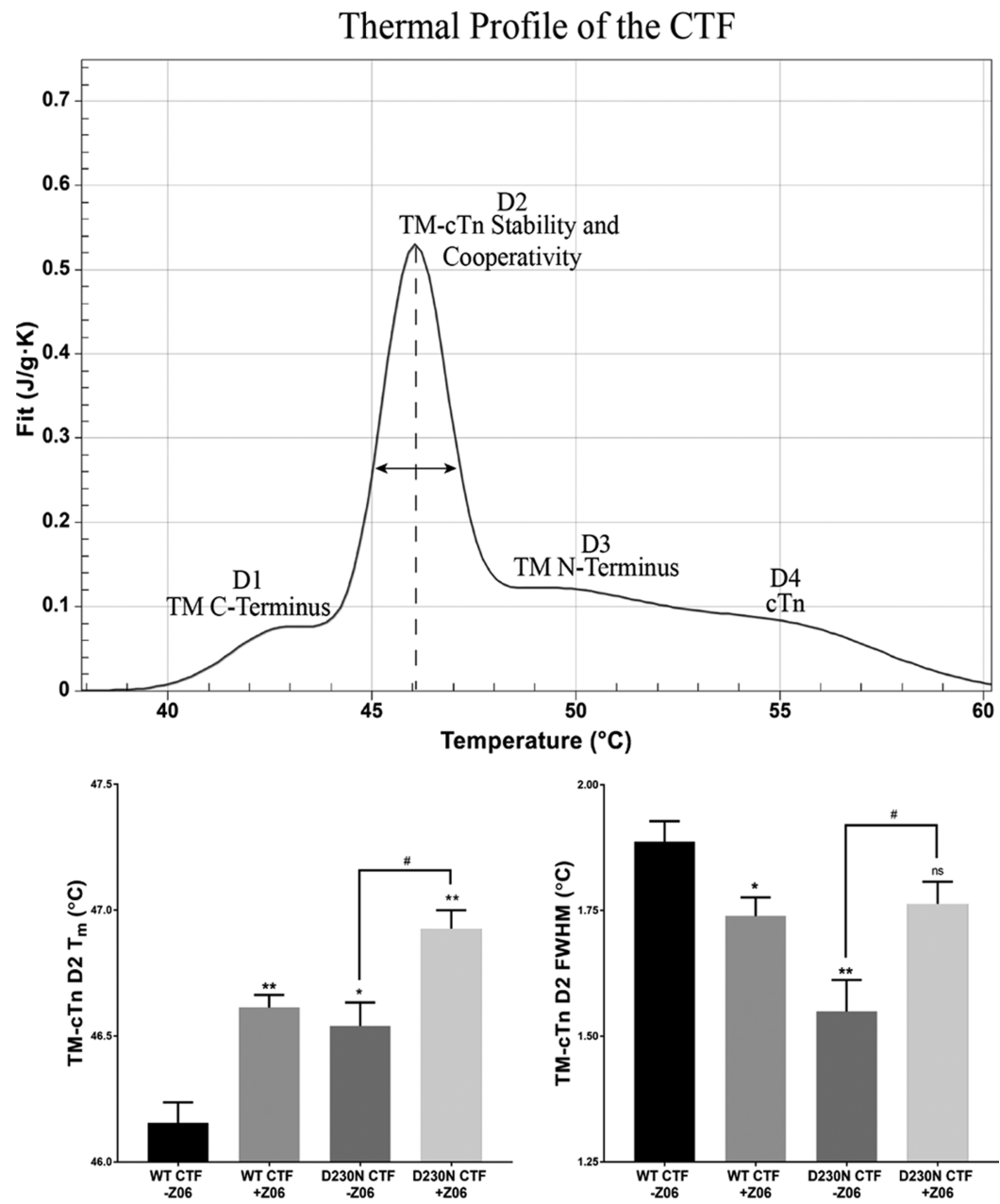

Figure 7. Results from DSC measurements of the WT thin filament, D230N mutant thin filament, and the mutant treated with the Z06 candidate drug. The peak of specific interest labeled "D2" is identified.

Table 2. Summary of Calorimetric Data Obtained via DSC ${ }^{a}$

\begin{tabular}{lll} 
& \multicolumn{1}{c}{$T_{\mathrm{m}}{ }^{\circ} \mathrm{C}$} & \multicolumn{1}{c}{ fwhm ${ }^{\circ} \mathrm{C}$} \\
WT CTF - Z06 & $46.2 \pm 0.08$ & $1.9 \pm 0.04$ \\
WT CTF + Z06 & $46.6 \pm 0.05^{* *}$ & $1.7 \pm 0.04^{*}$ \\
D230N CTF - Z06 & $46.5 \pm 0.09^{*}$ & $1.5 \pm 0.06^{* *}$ \\
D230N CTF + Z06 & $46.9 \pm 0.07^{* * * \#}$ & $1.8 \pm 0.04^{\#}$
\end{tabular}

${ }^{a}$ Thermal stability $\left(T_{\mathrm{m}}{ }^{\circ} \mathrm{C}\right)$ and cooperativity $\left(\right.$ fwhm, $\left.{ }^{\circ} \mathrm{C}\right)$ for $\mathrm{TM}$ WT and TM D230N bound to Z06 values are reported as mean \pm SEM for calorimetric domain 2 (D2) which corresponds to the thermal unfolding of the TM-cTn complex off of actin. There was an $N=3-4$ per group. A student's $t$-test was used to assess significance, ${ }^{*} p<0.05$ vs WT $-Z 06,{ }^{*} p<0.01$ vs WT $-Z 06$, and \#p $<0.05$ vs $\mathrm{D} 230 \mathrm{~N}-\mathrm{Z} 06$.

largely true for almost any drug candidate proposed. In addition, the individual methods used to study the effect of this small molecule could all be potentially subject to question. For example, the only atomic information we have on the drug binding is computational, and one may argue the molecule may be finding other binding sites. That having been said, both experimental methodologies point to the same conclusions as the computations - the drug binds and effects the thin filament in ways that are potentially therapeutic. The next step will be to test this and other candidate drugs in animal models. This higher level of biological complexity can be studied in transgenic animal models created in our group. In addition, other studies of binding - such as NMR could be used to verify the exact binding location.

\section{COMPUTATIONAL METHODS}

NAMD 2.9 software ${ }^{36}$ with the CHARMM27 force field was used to perform all MD simulations of the full atomistic thin filament systems. The last slice from the equilibration steps from earlier WT and TM D230N mutant structures ${ }^{7}$ was used as a starting point for docking studies. Schrödinger SiteMap $4.1^{28,37,38}$ was used to identify potential binding pockets within the cTn complex. For this purpose, the Cartesian coordinates of each atom from the cTnC subunit (residues 1-161), cTnI subunit (residues 1-210), cTnT subunit (residues 120-287), TM (residues 100-265), and calcium cations were imported into the Schrödinger Maestro software. ${ }^{39}$ Each chain was verified with the Protein Preparation Wizard ${ }^{40,41}$ to ensure that all amino acids are consistent with the corresponding residues from the initial NAMD PDB file. In the case of truncated segments (cTnT, TM), all $\mathrm{N}$-end and $\mathrm{C}$-end residues were converted to their appropriate form. Reduction of the size of the system was necessary because of the limit of number of atoms that can be processed by Schrödinger SiteMap. In addition, usage of TM chains prevents Schrödinger SiteMap from identifying potential binding pockets in the area where interactions between $\mathrm{CTnT}$ and $\mathrm{TM}$ are present. 
Schrödinger SiteMap was set-up to identify the top 20 binding pockets with fine grids and at least 15 site points for each reported binding pocket. As a result, 11-16 potential binding pockets were identified. For each system, the top ranked binding pocket we found was localized between $\mathrm{N}$ and C lobes of the cTnC subunit (see the examples of the Schrödinger SiteMap surfaces in the Supporting Information). Therefore, in Schrödinger Glide $7.3^{42,43}$ software, the whole cTnC subunit was chosen to generate a grid for further docking procedures.

The ZINC15 database ${ }^{30}$ has been chosen as a source of ligands that contains millions of possible drug candidates. From this, a large set of 3D ligand structures have been exported with the following criteria: molecular weight from $450 \mathrm{Da}$ to above $500+\mathrm{Da}$ (tranches: $450-500 \mathrm{Da}, 500+\mathrm{Da}$ ) and $\log \mathrm{P}$ values below 5 . The relatively high mass of ligands was chosen to be sure that molecules are big enough to not fit in small gaps between different chains but small enough to fit between cTnC lobes. Ligands from each tranche have been prepared for docking in Schrödinger LigPrep 4.0 software $^{44}$ with retention of chirality and with the generation of all tautomers, ring conformations, and possible ionization states by Schrödinger Epik 3.8 software $^{45-47}$ at $\mathrm{pH} 7.0 \pm 1.0$. After this preparation step, each ligand set has been subjected to a flexible high-throughput virtual screening (HTVS) docking in Schrödinger Glide software to the binding pocket within the TM D230N cTnC subunit. All docked ligands with Glide docking scores below -6.0 have been re-docked with the standard precision mode (SP) in Schrödinger Glide software to the same binding pocket. Finally, after SP docking procedures, ligands with Glide docking scores below -6.0 were re-docked with the extra precision mode $(\mathrm{XP})^{43}$ in Schrödinger Glide software. The set of ligands with the best $\mathrm{XP}$ docking scores were re-docked with the XP mode in the binding site within the $\mathrm{hTnC}$ chain opposite side to $\mathrm{cTnC}$ in CTF model. The ligands with the best average XP docking scores were selected for further MD simulations. In order to test the accuracy of our docking procedure, we blindly docked the known calcium sensitizer 2,4-difluorobiphenyl-4-yloxy acetic acid to the binding pocket we find and we compare RMSD of the top binding pose to that reported in the literature. $^{48}$ The resulting structure had an RMSD when comparing to the reported structure of $0.65 \AA$, indicating the viability of the method.

The best ligand structures were exported from Schrödinger Maestro to the pdb files and missing CHARMM parameters for chosen ligands were assigned from the Multipurpose AtomTyper for CHARMM (MATCH) web server. ${ }^{49}$ In the next step, ligands structures were merged with the corresponding CTF structures (form the last slice of the equilibration) and resolvated in Visual Molecular Dynamics (VMD 1.92). ${ }^{50}$ We briefly outline the prior MD methodology.

After solvation and addition of counter-ions, each system (comprised of roughly 5 million atoms) was minimized for 5000 steps with periodic boundary conditions, particle mesh Ewald summation for electrostatics, SHAKE for bonds involving hydrogen, and with van der Waals interactions cut off at $12 \AA$. Each model was heated from 0 to $300 \mathrm{~K}$ with a temperature increment of $1 \mathrm{~K} / \mathrm{ps}$ and then simulated for additional $10 \mathrm{ps}$ to ensure that the temperature is stable at 300 K. Next, 690 ps of equilibration with isothermal-isobaric conditions was performed (Langevin piston set at 1 atm, Langevin thermostat set at $300 \mathrm{~K}$ ). In the final step, three separate $10 \mathrm{~ns} \mathrm{MD}$ productions runs were performed with the same conditions as in the equilibration step but with different randomly chosen velocity initialization seeds. The average structures were created in the VMD1.92 and re-minimized for 5000 steps in the continuum solvation model (water) in NAMD. RMSF calculations and hydrogen bond counts were performed in VMD1.92. Average distances between TM and TnT were calculated as the average of distances between fictitious beads located at the center of mass of each helix. The distance between $\mathrm{cTnT}$ and the two tropomyosin chains was averaged.

We note that the starting structure can significantly influence $\mathrm{MD}$ results. As such, the three independent $\mathrm{MD}$ runs are intended to provide a check on outlier results. While we have found such results in some of our past studies, all results reported here are from the initial three runs and are selfconsistent.

\section{EXPERIMENTAL MATERIALS AND METHODS}

Protein Expression, Purification, and Reconstitution. Alanine-serine-tagged $\alpha$-tropomyosin were expressed and purified as previously described. ${ }^{3,4}$ The ala-ser tag is used to mimic N-terminal acetylation and is necessary to promote head-to-tail polymerization binding with actin and Tn. ${ }^{51}$ The D230N mutation was inserted into the WT TM pET3D vector (provided by Dr. Wieczorek) via QuikChange site-directed mutagenesis (Stratagene). Human cardiac troponin proteins were expressed and purified, and the recombinant cTn complex reconstitution was performed in equimolar amounts as previously described. ${ }^{52}$ After reconstitution, $\mathrm{TnT}$ precipitate was separated via centrifugation at $14000 \mathrm{rpm}$ for $20 \mathrm{~min}$. Rabbit skeletal muscle was used to purify, prepare, and polymerize filamentous actin as previously described in the literature. ${ }^{53}$ G-actin was then extracted from the acetone powder and prior to reconstitution of the thin filaments, Gactin was polymerized to $\mathrm{F}$-actin with a final salt concentration of $50 \mathrm{mM} \mathrm{KCl}$ and $2 \mathrm{mM} \mathrm{MgCl}_{2}$. To ensure complete polymerization actin was thoroughly mixed and polymerized at room temperature for $1 \mathrm{~h}$. Individual proteins were dialyzed in their appropriate buffer systems ( $\mathrm{X} 3$ change, $>4 \mathrm{~h}$ ), and protein concentrations were measured using a Beckman Coulter DU-730 UV-vis spectrophotometer (extinction coefficients were determined by the primary sequence of the protein). Dialyzed proteins were reconstituted into thin filaments at a 3:3:7 $\mathrm{Tn} / \mathrm{Tm} /$ actin molar ratio. The reconstituted thin filaments were incubated on ice overnight prior to use.

Isothermal Titration Calorimetry. ITC measurements were performed with a Nano ITC ultrasensitive titration calorimeter (TA Instruments). Prior to reconstitution, thin filament proteins were dialyzed into a buffer containing $10 \mathrm{mM}$ MOPS, $140 \mathrm{mM} \mathrm{KCl}, 50 \mu \mathrm{M} \mathrm{CaCl}_{2}, 7 \mathrm{mM} \mathrm{MgCl}_{2}, 1 \mathrm{mM}$ $\mathrm{NaN}_{3}$, and $100 \mu \mathrm{M}$ DTT, pH 7.35. Z01 and Z06 ligands were brought up in ITC Buffer to a final concentration of 250 and $500 \mu \mathrm{M}$, respectively. A $2 \mu \mathrm{L}$ injection of drug (titrant) was delivered (30 injections) to the reaction cell over with a $350 \mathrm{~s}$ interval between injections to allow complete equilibration. The cell was stirred at $75 \mathrm{rpm}$ to ensure rapid mixing and to minimize protein shearing. The temperature was maintained at $20{ }^{\circ} \mathrm{C}$. Raw traces for each system were collected in quadruplicate. Traces were analyzed via NanoAnalyze (TA Instruments). A titrant into buffer only was run to account for the heat of dilution and was in agreement with the last 
experimental injections at saturation. Thus, the average heat of the final (5) injections was used to correct for the heat of dilution. The heats of each experimental injection were integrated and normalized to titrant and then plotted against the molar ratio of the drug added to TF. TF concentrations were calculated based on the total protein concentration and Tn complex concentration obtained via UV spectrophotometry. The fitted curve provides a thermodynamic description of the binding interaction via binding affinity $\left(K_{\mathrm{d}}\right)$ and the enthalpy of binding $(\Delta H)$.

Differential Scanning Calorimetry. A Nano DSC (TA Instruments) was used to assay the thermal stability (or calorimetric profile) of fully reconstituted CTFs (actin/Tm/ cTn). The individual proteins were dialyzed into a buffer containing $30 \mathrm{mM}$ HEPES, $200 \mathrm{mM} \mathrm{KCl}, 50 \mu \mathrm{M} \mathrm{CaCl}_{2}, 1 \mathrm{mM}$ $\mathrm{MgCl}_{2}, 200 \mu \mathrm{M} \mathrm{Mg-ATP}$, and $1 \mathrm{mM} \beta \mathrm{ME}, \mathrm{pH} 7.35$. Thin filaments were reconstituted in accordance with previous work by Kremneva et al. ${ }^{32}$ For each replicate, $600 \mu \mathrm{L}$ of protein (CTF total protein concentration between 1.85 and $1.9 \mathrm{mg} /$ $\mathrm{mL}$ ) was loaded into the sample cell and heated from 25 to 75 ${ }^{\circ} \mathrm{C}$ at a rate of $1{ }^{\circ} \mathrm{C} / \mathrm{min}$ after an initial $600 \mathrm{~s}$ equilibration period $(n=3)$. A buffer versus buffer scan was also performed daily and applied to background. The resultant calorimetric heating profile was fitted via a 4-Gaussian distribution and analyzed using NanoAnalyze software provided by TA Instruments.

\section{ASSOCIATED CONTENT}

\section{S Supporting Information}

The Supporting Information is available free of charge on the ACS Publications website at DOI: 10.1021/acsomega.8b03340.

Table S1: Comparison of available PDB structures of cTnC subunit with drug molecules and PDB structures used in drug design studies, Figure S1: overlap of known PDB structures of C-lobe cTnC region with drugs or used in drug design studies, Figure S2: overlap of known PDB structures of $\mathrm{N}$-lobe $\mathrm{cTnC}$ region with drugs to the last slice from the equilibration of $\mathrm{D} 230 \mathrm{~N}$ mutant, Figure S3: overlap of known PDB structures by Takeda et al. to the last slice from the equilibration of $\mathrm{D} 230 \mathrm{~N}$ mutant Figure S4: cTnC and cTnI structures, Table S2: example result of the Schrödinger SiteMap binding pocket analysis of the cTn complex, Figure S5: example of the hydrophobic (yellow), donor (blue) and acceptor (red) maps within the binding pocket, Figure S6: comparison of the histograms of distances between the center of the masses of $\mathrm{C} \alpha$ atoms of $c \operatorname{TnT}$ chain (residues 94 to 133) and TM chains, Figure S7: comparison of the histograms of distances between the center of the masses of $\mathrm{C} \alpha$ atoms of $\mathrm{CTnT}$ chain (residues 94 to 133) and TM chains with Z06, Figure S8: comparison of the histograms of distances between the center of the masses of $\mathrm{C} \alpha$ atoms of cTnT chain (residues 94-133) and TM chains with Z08, Figure S9: $\mathrm{C} \alpha$ RMSF plots for cTnC subunit for WT, D230N, D230N-Z06 and WT-Z06, Figure S10: C $\alpha$ RMSF plots for cTnI subunit for WT, D230N, D230N-Z06 and WTZ06, Figure S11: C $\alpha$ RMSF plots for cTnT subunit for WT, D230N, D230N-Z06 and WT-Z06, Figure S.11: $\mathrm{C} \alpha$ RMSF plots for cTnT subunit for WT, D230N, D230N-Z06 and WT-Z06, Figure S.12: CHARMM atoms name for Asp, Glu, Lys and Arg. Heavy atoms are colored in green, Figure S.13: C $\alpha$ RMSF values for 11 overlapping TMC-TMN residues plus 15 additional residues close to the TMC-TMN overlap region for WT, TM D230N mutant and TM D230N-Z01 systems, Figure S.14: C $\alpha$ RMSF plots for cTnC subunit for WT, D230N, D230N-Z01, Figure S15: C $\alpha$ RMSF plots for cTnI subunit for WT, D230N, D230N-Z01. Figure S16: $\mathrm{C} \alpha$ RMSF plots for cTnI subunit for WT, D230N, D230N-Z01, Figure S.17: RMSF values for heavy atoms of most important cTnC and $\mathrm{cTnI}$ residues (PDF)

\section{AUTHOR INFORMATION}

\section{Corresponding Author}

*E-mail: sschwartz@email.arizona.edu.

ORCID

Steven D. Schwartz: 0000-0002-0308-1059

Notes

The authors declare no competing financial interest.

\section{ACKNOWLEDGMENTS}

This work supported by the NIH through grant HL136375 to SDS and JCT.

\section{ABBREVIATIONS}

CTF, cardiac thin filament; TM, tropomyosin; TMC, C-end of the tropomyosin chain; TMN, N-end of the tropomyosin chain; cTnC, troponin C; cTnI, troponin I; cTnT, troponin T; cTnI-IR, inhibitory region of troponin I; $\mathrm{MD}$, molecular dynamics; RMSF, root mean square fluctuation; WT, wild type; TM D230N, tropomyosin Asp230Asn mutant; TM D230N-Z01/TM D230N-Z06/TM D230N-Z08, tropomyosin Asp230Asn cardiac thin filament mutant with Z01/Z06/Z08 ligand bound; WT-Z01/WT-Z06/WT-Z08, wild type cardiac thin filament with $Z 01 / Z 06 / Z 08$ ligand bound

\section{REFERENCES}

(1) Tardiff, J. C. Thin Filament Mutations. Circ. Res. 2011, 108, $765-782$.

(2) Thierfelder, L.; Watkins, H.; MacRae, C.; Lamas, R.; McKenna, W.; Vosberg, H.-P.; Seldman, J. G.; Seidman, C. E. $\alpha$-tropomyosin and cardiac troponin $\mathrm{T}$ mutations cause familial hypertrophic cardiomyopathy: A disease of the sarcomere. Cell 1994, 77, 701-712.

(3) Geisterfer-Lowrance, A. A. T.; Kass, S.; Tanigawa, G.; Vosberg, H.-P.; McKenna, W.; Seidman, C. E.; Seidman, J. G. A molecular basis for familial hypertrophic cardiomyopathy: A $\beta$ cardiac myosin heavy chain gene missense mutation. Cell 1990, 62, 999-1006.

(4) Rohde, J. A.; Thomas, D. D.; Muretta, J. M. Heart failure drug changes the mechanoenzymology of the cardiac myosin powerstroke. Proc. Natl. Acad. Sci. U.S.A. 2017, 114, E1796-E1804.

(5) Green, E. M.; Wakimoto, H.; Anderson, R. L.; Evanchik, M. J.; Gorham, J. M.; Harrison, B. C.; Henze, M.; Kawas, R.; Oslob, J. D.; Rodriguez, H. M.; Song, Y.; Wan, W.; Leinwand, L. A.; Spudich, J. A.; McDowell, R. S.; Seidman, J. G.; Seidman, C. E. A small-molecule inhibitor of sarcomere contractility suppresses hypertrophic cardiomyopathy in mice. Science 2016, 351, 617-621.

(6) Kawas, R. F.; Anderson, R. L.; Ingle, S. R. B.; Song, Y.; Sran, A. S.; Rodriguez, H. M. A small-molecule modulator of cardiac myosin acts on multiple stages of the myosin chemomechanical cycle. J. Biol. Chem. 2017, 292, 16571-16577.

(7) McConnell, M.; Tal Grinspan, L.; Williams, M. R.; Lynn, M. L.; Schwartz, B. A.; Fass, O. Z.; Schwartz, S. D.; Tardiff, J. C. Clinically Divergent Mutation Effects on the Structure and Function of the 
Human Cardiac Tropomyosin Overlap. Biochemistry 2017, 56, 34033413.

(8) Williams, M. R.; Lehman, S. J.; Tardiff, J. C.; Schwartz, S. D. Atomic resolution probe for allostery in the regulatory thin filament. Proc. Natl. Acad. Sci. U.S.A. 2016, 113, 3257-3262.

(9) Jin, J.-P.; Chong, S. M. Localization of the two tropomyosinbinding sites of troponin T. Arch. Biochem. Biophys. 2010, 500, 144150.

(10) Lynn, M. L.; Tal Grinspan, L.; Holeman, T. A.; Jimenez, J.; Strom, J.; Tardiff, J. C. The structural basis of alpha-tropomyosin linked (Asp230Asn) familial dilated cardiomyopathy. J. Mol. Cell. Cardiol. 2017, 108, 127-137.

(11) Lakdawala, N. K.; Dellefave, L.; Redwood, C. S.; Sparks, E.; Cirino, A. L.; Depalma, S.; Colan, S. D.; Funke, B.; Zimmerman, R. S.; Robinson, P.; Watkins, H.; Seidman, C. E.; Seidman, J. G.; McNally, E. M.; Ho, C. Y. Familial Dilated Cardiomyopathy Caused by an Alpha-Tropomyosin Mutation. J. Am. Coll. Cardiol. 2010, 55, 320329.

(12) Yar, S.; Chowdhury, S. A. K.; Davis, R. T.; Kobayashi, M.; Monasky, M. M.; Rajan, S.; Wolska, B. M.; Gaponenko, V.; Kobayashi, T.; Wieczorek, D. F.; Solaro, R. J. Conserved Asp-137 Is Important for both Structure and Regulatory Functions of Cardiac $\alpha$ Tropomyosin $(\alpha$-TM) in a Novel Transgenic Mouse Model Expressing $\alpha$-TM-D137L. J. Biol. Chem. 2013, 288, 16235-16246.

(13) Sumida, J. P.; Wu, E.; Lehrer, S. S. Conserved Asp-137 imparts flexibility to tropomyosin and affects function. J. Biol. Chem. 2008, 283, 6728-6734.

(14) Li, X.; Suphamungmee, W.; Janco, M.; Geeves, M. A.; Marston, S. B.; Fischer, S.; Lehman, W. The flexibility of two tropomyosin mutants, D175N and E180G, that cause hypertrophic cardiomyopathy. Biochem. Biophys. Res. Commun. 2012, 424, 493-496.

(15) Li, M. X.; Hwang, P. M. Structure and function of cardiac troponin C (TNNC1): Implications for heart failure, cardiomyopathies, and troponin modulating drugs. Gene 2015, 571, 153-166.

(16) Lindert, S.; Li, M. X.; Sykes, B. D.; McCammon, J. A. Computer-Aided Drug Discovery Approach Finds Calcium Sensitizer of Cardiac Troponin. Chem. Biol. Drug Des. 2015, 85, 99-106.

(17) Wang, X.; Li, M. X.; Sykes, B. D. Structure of the Regulatory Ndomain of Human Cardiac Troponin $\mathrm{C}$ in Complex with Human Cardiac Troponin I147-163and Bepridil. J. Biol. Chem. 2002, 277, 31124-31133.

(18) Oleszczuk, M.; Robertson, I. M.; Li, M. X.; Sykes, B. D. Solution structure of the regulatory domain of human cardiac troponin $\mathrm{C}$ in complex with the switch region of cardiac troponin I and W7: The basis of W7 as an inhibitor of cardiac muscle contraction. J. Mol. Cell. Cardiol. 2010, 48, 925-933.

(19) Pineda-Sanabria, S. E.; Robertson, I. M.; Sun, Y.-B.; Irving, M.; Sykes, B. D. Probing the mechanism of cardiovascular drugs using a covalent levosimendan analog. J. Mol. Cell. Cardiol. 2016, 92, 174184.

(20) Lindhout, D. A.; Sykes, B. D. Structure and dynamics of the Cdomain of human cardiac troponin $\mathrm{C}$ in complex with the inhibitory region of human cardiac troponin I. J. Biol. Chem. 2003, 278, 2702427034.

(21) Wang, X.; Li, M. X.; Spyracopoulos, L.; Beier, N.; Chandra, M.; Solaro, R. J.; Sykes, B. D. Structure of the C-domain of Human Cardiac Troponin $\mathrm{C}$ in Complex with the Ca2+Sensitizing Drug EMD 57033. J. Biol. Chem. 2001, 276, 25456-25466.

(22) Li, Y.; Love, M. L.; Putkey, J. A.; Cohen, C. Bepridil opens the regulatory N-terminal lobe of cardiac troponin C. Proc. Natl. Acad. Sci. U.S.A. 2000, 97, 5140-5145.

(23) Pineda-Sanabria, S. E.; Robertson, I. M.; Sykes, B. D. Structure oftrans-Resveratrol in Complex with the Cardiac Regulatory Protein Troponin C. Biochemistry 2011, 50, 1309-1320.

(24) Li, M. X.; Robertson, I. M.; Sykes, B. D. Interaction of cardiac troponin with cardiotonic drugs: A structural perspective. Biochem. Biophys. Res. Commun. 2008, 369, 88-99.
(25) Brixius, K.; Reicke, S.; Schwinger, R. H. G. Beneficial effects of the Ca2+ sensitizer levosimendan in human myocardium. Am. J. Physiol.: Heart Circ. Physiol. 2002, 282, H131-H137.

(26) Cai, F.; Li, M. X.; Pineda-Sanabria, S. E.; Gelozia, S.; Lindert, S.; West, F.; Sykes, B. D.; Hwang, P. M. Structures reveal details of small molecule binding to cardiac troponin. J. Mol. Cell. Cardiol. 2016, 101, 134-144.

(27) Pollesello, P.; Ovaska, M.; Kaivola, J.; Tilgmann, C.; Lundstrom, K.; Kalkkinen, N.; Ulmanen, I.; Nissinen, E.; Taskinen, J. Binding of a New Ca2+ Sensitizer, Levosimendan, to Recombinant Human Cardiac Troponin-C - a Molecular Modeling, Fluorescence Probe, and Proton Nuclear-Magnetic-Resonance Study. J. Biol. Chem. 1994, 269, 28584-28590.

(28) Halgren, T. A. Identifying and Characterizing Binding Sites and Assessing Druggability. J. Chem. Inf. Model. 2009, 49, 377-389.

(29) Friesner, R. A.; Banks, J. L.; Murphy, R. B.; Halgren, T. A.; Klicic, J. J.; Mainz, D. T.; Repasky, M. P.; Knoll, E. H.; Shelley, M.; Perry, J. K.; Shaw, D. E.; Francis, P.; Shenkin, P. S. Glide: A new approach for rapid, accurate docking and scoring. 1. Method and assessment of docking accuracy. J. Med. Chem. 2004, 47, 1739-1749.

(30) Sterling, T.; Irwin, J. J. ZINC 15 - Ligand Discovery for Everyone. J. Chem. Inf. Model. 2015, 55, 2324-2337.

(31) Kuo, F.; Gillespie, T. A.; Kulanthaivel, P.; Lantz, R. J.; Ma, T. W.; Nelson, D. L.; Threlkeld, P. G.; Wheeler, W. J.; Yi, P.; Zmijewski, M. Synthesis and biological activity of some known and putative duloxetine metabolites. Bioorg. Med. Chem. Lett. 2004, 14, 34813486.

(32) Kremneva, E. V.; Nikolaeva, O. P.; Gusev, N. B.; Levitsky, D. I. Effects of troponin on thermal unfolding of actin-bound tropomyosin. Biochemistry 2003, 68, 802-809.

(33) Yar, S.; Chowdhury, S. A. K.; Davis, R. T., 3rd; Kobayashi, M.; Monasky, M. M.; Rajan, S.; Wolska, B. M.; Gaponenko, V.; Kobayashi, T.; Wieczorek, D. F.; Solaro, R. J. Conserved Asp-137 Is Important for both Structure and Regulatory Functions of Cardiac $\alpha$ Tropomyosin $(\alpha-\mathrm{TM})$ in a Novel Transgenic Mouse Model Expressing $\alpha$-TM-D137L. J. Biol. Chem. 2013, 288, 16235-16246.

(34) Kremneva, E.; Boussouf, S.; Nikolaeva, O.; Maytum, R.; Geeves, M. A.; Levitsky, D. I. Effects of Two Familial Hypertrophic Cardiomyopathy Mutations in $\alpha$-Tropomyosin, Asp175Asn and Glu180Gly, on the Thermal Unfolding of Actin-Bound Tropomyosin. Biophys. J. 2004, 87, 3922-3933.

(35) Niesen, F. H.; Berglund, H.; Vedadi, M. The use of differential scanning fluorimetry to detect ligand interactions that promote protein stability. Nat. Protoc. 2007, 2, 2212-2221.

(36) Phillips, J. C.; Braun, R.; Wang, W.; Gumbart, J.; Tajkhorshid, E.; Villa, E.; Chipot, C.; Skeel, R. D.; Kalé, L.; Schulten, K. Scalable molecular dynamics with NAMD. J. Comput. Chem. 2005, 26, 17811802.

(37) Schrödinger SiteMap 3.8; Schrödinger, LLC: New York, NY, 2017.

(38) Halgren, T. New method for fast and accurate binding-site identification and analysis. Chem. Biol. Drug Des. 2007, 69, 146-148.

(39) Schrödinger Release 2017-4; Maestro, Schrödinger, LLC: New York, NY, 2017.

(40) 2017 Schrödinger Release 2017-4: Schrödinger Suite 2017-4 Protein Preparation Wizard; Epik, Schrödinger, LLC: New York, NY, 2016; Impact, Schrödinger, LLC, New York, NY, 2016; Prime, Schrödinger, LLC, New York, NY.

(41) Sastry, G. M.; Adzhigirey, M.; Day, T.; Annabhimoju, R.; Sherman, W. Protein and ligand preparation: parameters, protocols, and influence on virtual screening enrichments. J. Comput. Aided. Mol. Des. 2013, 27, 221-234.

(42) Schrödinger Glide 7.3; Schrödinger, LLC: New York, NY, 2017.

(43) Friesner, R. A.; Murphy, R. B.; Repasky, M. P.; Frye, L. L.; Greenwood, J. R.; Halgren, T. A.; Sanschagrin, P. C.; Mainz, D. T. Extra Precision Glide: Docking and Scoring Incorporating a Model of Hydrophobic Enclosure for Protein-Ligand Complexes. J. Med. Chem. 2006, 49, 6177-6196. 
(44) Schrödinger Release 2017-4: LigPrep 4.0; Schrödinger, LLC: New York, NY, 2017.

(45) Schrödinger Release 2017-4: Epik 3.8; Schrödinger, LLC: New York, NY, 2017.

(46) Greenwood, J. R.; Calkins, D.; Sullivan, A. P.; Shelley, J. C. Towards the comprehensive, rapid, and accurate prediction of the favorable tautomeric states of drug-like molecules in aqueous solution. J. Comput.-Aided Mol. Des. 2010, 24, 591-604.

(47) Shelley, J. C.; Cholleti, A.; Frye, L. L.; Greenwood, J. R.; Timlin, M. R.; Uchimaya, M. Epik: a software program for $\mathrm{pK}$ a prediction and protonation state generation for drug-like molecules. $J$. Comput.-Aided Mol. Des. 2007, 21, 681-691.

(48) Robertson, I. M.; Sun, Y.-B.; Li, M. X.; Sykes, B. D. A structural and functional perspective into the mechanism of $\mathrm{Ca} 2+$-sensitizers that target the cardiac troponin complex. J. Mol. Cell. Cardiol. 2010, 49, 1031-1041.

(49) Yesselman, J. D.; Price, D. J.; Knight, J. L.; Brooks, C. L. MATCH: An Atom-Typing Toolset for Molecular Mechanics Force Fields. J. Comput. Chem. 2012, 33, 189-202.

(50) Humphrey, W.; Dalke, A.; Schulten, K. VMD: Visual molecular dynamics. J. Mol. Graph. Model. 1996, 14, 33-38.

(51) Monteiro, P. B.; Lataro, R. C.; Ferro, J. A.; Reinach Fde, C. Functional alpha-tropomyosin produced in Escherichia coli. A dipeptide extension can substitute the amino-terminal acetyl group. J. Biol. Chem. 1994, 269, 10461-10466.

(52) Sumandea, M. P.; Pyle, W. G.; Kobayashi, T.; de Tombe, P. P.; Solaro, R. J. Identification of a functionally critical protein kinase C phosphorylation residue of cardiac troponin T. J. Biol. Chem. 2003, 278, 35135-35144.

(53) Spudich, J. A.; Watt, S. The regulation of rabbit skeletal muscle contraction. I. Biochemical studies of the interaction of the tropomyosin-troponin complex with actin and the proteolytic fragments of myosin. J. Biol. Chem. 1971, 246, 4866-4871. 